\title{
Supporting Info to: A theoretical study on the factors influencing cyanine photoisomerization: the case of thiacyanine in gas phase and in methanol
}

\author{
A contribution from \\ R. Improta ${ }^{a}$, F. Santoro ${ }^{b}$ \\ ${ }^{a}$ Istituto di Biostrutture e Biommagini del CNR, via Mezzocannone 6, I-80134 \\ Napoli, Italy. ${ }^{b}$ Istituto per i Processi Chimico-Fisici del CNR, Area della Ricerca \\ del CNR di Pisa, via Moruzzi 1, I-56124 Pisa, Italy
}

\section{Results on stCY}

We applied the computational approach described in Section 2 to the study of stCY. We calculated the TD-PBE0/6-31G(d) energies of the most significant stationary points determined at the CASSCF/6-31G(d) level on the $S_{0}$ and the $S_{1}$ potential energy surfaces. ${ }^{29}$ The results are reported in Table S1 and Figure S1 (see chart 1 for atom labelling). On the ground state $S_{0} \mathrm{PBE} 0$ predicts an equilibrium geometry very close to the CASSCF one. The two computed vertical excitation energies are close, the TD-PBE0 results being lower and closer to the experimental value (4.36 $\mathrm{eV})$. The quality of the comparison is unaltered at the CAS symmetric $\left(C_{2 v}\right.$ and $\left.C_{2}\right) S_{1}$ stationary points where TD-PBE0/6-31G(d) energies are lower and always within $0.3 \mathrm{eV}$ with respect to the CASMP2 ones; the agreement is still better on the $S_{1}-S_{0}$ energy gap (within $0.23 \mathrm{eV}$ ). The agreement is still good at the $C_{2}$ conical intersection, while TD-PBE0 does not converge at the asymmetric $C_{1}$ CI. This result indirectly confirms that in that region of the PES $S_{0}$ and $S_{1}$ are extremely close in energy according to TD-PBE0 calculations as well. It is indeed well known that Time Dependent Density Functional Theory rigorously holds for non-degenerate states only.

As expected TD-PBE0/6-31G(d) performs worse, at the $C_{1}$ global minimum, 
where the $S_{0} \rightarrow S_{1}$ transition has a charge-transfer character. In fact TD-DFT is known usually to underestimate the energy associated to this kind of interaction (ref. [41]), and it indeed provides an $S_{1}-S_{0}$ energy gap $\approx 0.8 \mathrm{eV}$ lower than the CASMP2 value.

The qualitative description of the PES obtained by performing CIS/6-31G(d) $S_{1}$ geometry optimizations is in reasonable agreement with that obtained at the CASSCF level. In particular, CIS optimization locates a $C_{2}$ local minimum with an almost perpendicular configuration, $\phi=\phi^{\prime}=110^{\circ}$ at $\mathrm{CAS}$ level and $=98^{\circ}$ at CIS level, whose $S_{1}$ energy is still in nice agreement with CASMP2. At the same geometry $S_{0}$ appears to be over-destabilized and gets too close to the $S_{1}$ value as a result of the structural differences with respect to the CAS structure.

The same qualitative agreement is found at $S_{1}$-Min, the $S_{1}$ global $C_{1}$ minimum for which both methods predicts a TICT character, where one of the two side-chains is coplanar with the central C-H bond while the other is almost perpendicular $\left(76^{\circ}\right.$ and $86^{\circ}$ at the CASSCF and CIS level, respectively). However, the TD-PBE0/6-31G(d) energy of the $S_{0}$ and $S_{1}$ states are quite different when using the CIS or CASSCF geometries. This result is due to differences in geometries, mainly in the value of the $\mathrm{C}_{0}-\mathrm{C}_{2}$ and $\mathrm{C}_{0}-\mathrm{C}_{2}^{\prime}$ bonds.

However, notwithstanding these discrepancies, it is worth highlighting that the qualitative description of the $S_{1}$ PES obtained at the TD-PBE0/6-31G(d)//CIS/6-31G(d) level is very similar to that provided by CASMP2 calculation: on the $S_{1}$ PES there is a barrierless motion driving towards a perpendicular TICT minimum. It is interesting to notice that CIS optimization underestimates but reproduces qualitatively a significant pyramidalization at the nitrogen $\mathrm{N}_{3}^{\prime}$ of the perpendicular moiety. This is of relevance since, as discussed in the article, one of the main differences between the global minimum of $\mathrm{H}-\mathrm{TCY}$ and stCY is actually the hybridization of $\mathrm{N}_{3}^{\prime}\left(s p^{2}\right.$ and almost $s p^{3}$, respectively). This difference makes the conical intersection $C_{1}$-CI much closer to the global minimum $S_{1}-\mathrm{Min} C_{1}$ in stCY than in x-TCY, suggesting a faster nonadiabatic transition for stCY.

From the quantitative point of view, the closest the system is to the planarity the more accurate are the results of our approach, that is instead expected to un- 
derestimate the $S_{1}-S_{0}$ gaps of the TICT pseudoperpendicular states.

The similarity of the physical picture provided for stCY by CASMP2 and TDPBEO//CIS calculations is comforting. The absorption spectra of unsubstutited streptocyanines are known indeed as one of the case in which TD-DFT exhibits the worst performances, over-estimating (as CASMP2) the excitation energy by more than $1 \mathrm{eV}$. The estimate provided by CASPT2/ANO calculations (see Schreiber, M.; Buß, V.; Fülscher, M. Phys. Chem. Chem. Phys. 2001, 3, 3906.) is much closer to the experimental results, but, unfortunately, the study of Schreiber et al. do not investigate the $S_{1}$ dynamics of stCY. 
Figure S1: Comparison of CASMP2/CASSCF, TD-PBE0/CASSCF and TDPBE0/CIS computed energies of different stationary points on the $\mathrm{S}_{1}$ state PES of stCY. The basis set is $6-31 \mathrm{G}(\mathrm{d})$.

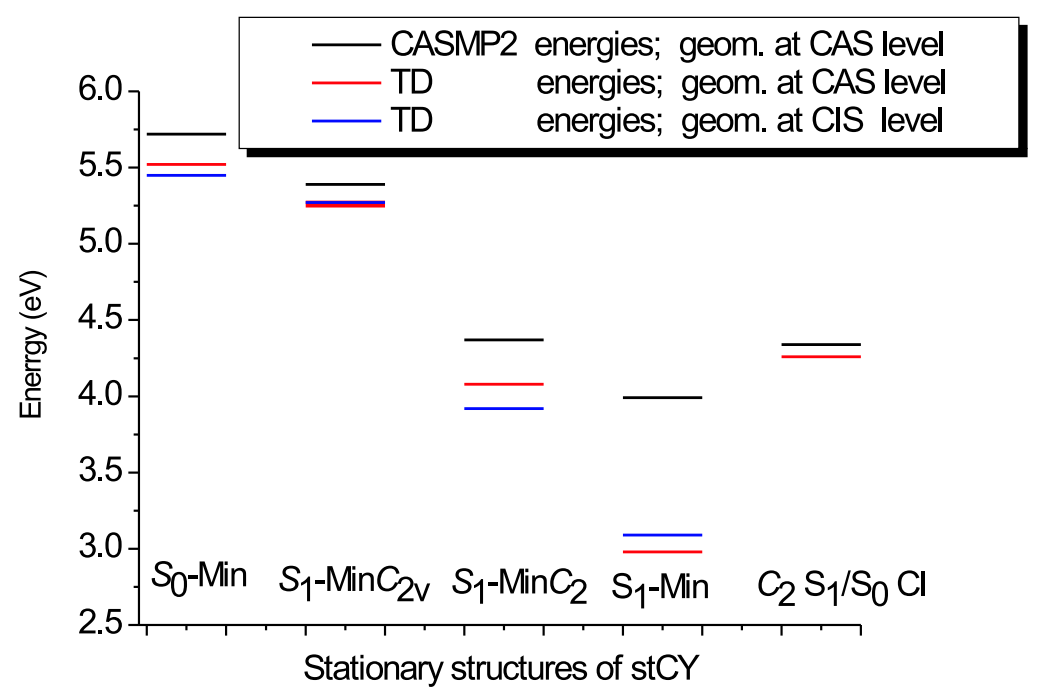


Figure S2: Total Dipole moment (in Debye) and Electrostatic solvation energy (in eV) of the $\mathrm{S}_{1}$ state of H-TCY as a function of the $\phi^{\prime}$ dihedral (in degrees). PCM/CIS/6-31G(d) calculations on the structures of the $\mathrm{cP}$ curve.

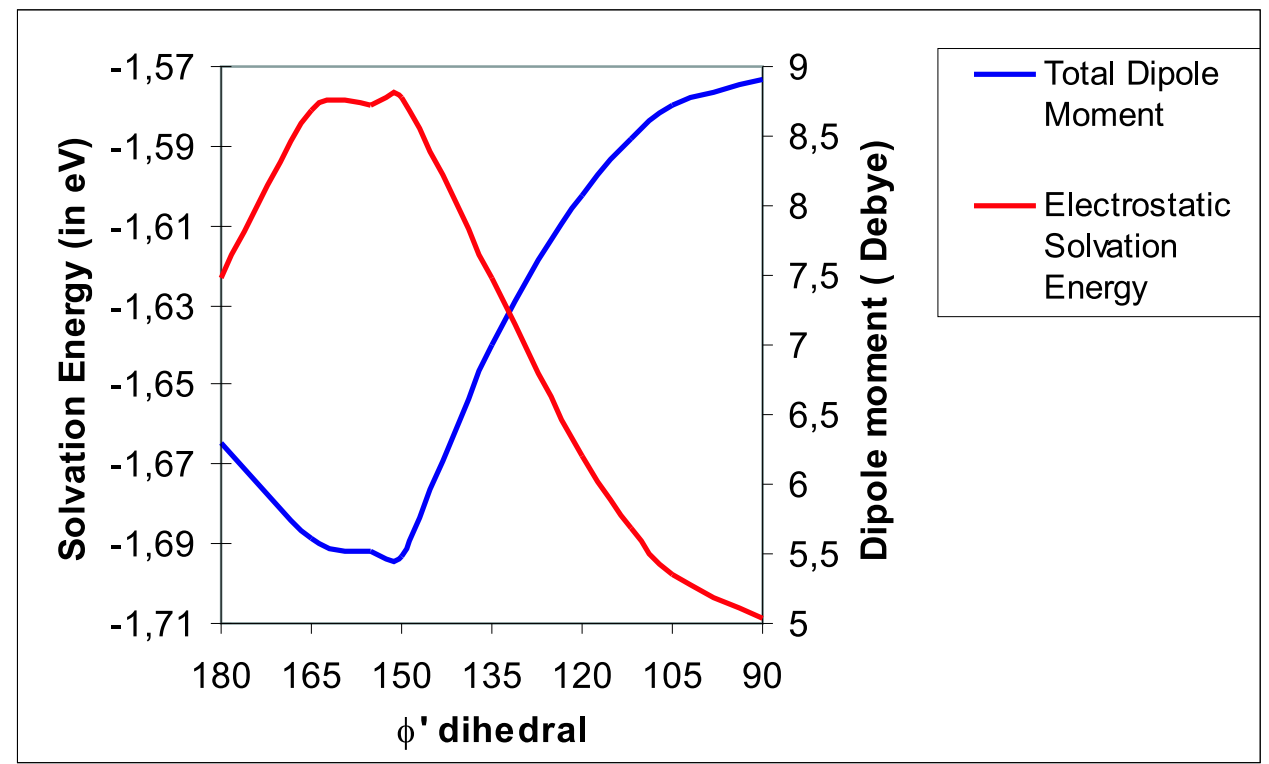


Table S1: Comparison of CASMP2/CASSCF, TD-PBE0/CASSCF and TD-PBE0/CIS results on stCY. The basis set is $6-31 \mathrm{G}(\mathrm{d})$. Energies in $\mathrm{eV}$, bond lengths in angstroms, angles in degrees.CASSCF/CASMP2 Results are taken from ref. [29]

\begin{tabular}{c|c|c|c|c|c|c|c|c|c}
\hline & \multicolumn{4}{|c|}{ Structural parameters } & \multicolumn{4}{c}{${ }^{a}$ Energies } \\
\hline & $\mathrm{C}_{0}-\mathrm{C}_{2}$ & $\mathrm{C}_{2}-\mathrm{N}_{3}$ & $\beta^{b}$ & $\gamma^{c}$ & ${ }^{d} \Omega N_{3}$ & ${ }^{d} \phi$ & CASSCF & TD-PBE0 \\
& $\left(\mathrm{C}_{0}-\mathrm{C}_{2}^{\prime}\right)$ & $\left(\mathrm{C}_{2}^{\prime}-\mathrm{N}_{3}^{\prime}\right)$ & & ${ }^{c}\left(\gamma^{\prime}\right)$ & $d\left(\Omega N_{3}^{\prime}\right)$ & ${ }^{e}\left(\phi^{\prime}\right)$ & $(\mathrm{CASMP})$ & \\
& & & $S_{0}$ & $S_{1}$ & $S_{0}$ & $S_{1}$ \\
\hline
\end{tabular}

CASSCF

Geometries

\begin{tabular}{c|c|c|c|c|c|c|c|c|c|c}
\hline $\boldsymbol{S}_{0}$-trans & 1.386 & 1.308 & 119.0 & 125.6 & 360.0 & 180.0 & $\mathbf{0 . 0 0}$ & 5.44 & 0.08 & 5.57 \\
& 1.386 & 1.308 & & 125.6 & 360.0 & 180.0 & $\mathbf{0 . 0 0}$ & 5.72 & & \\
\hline $\boldsymbol{S}_{1}$-Min $\boldsymbol{C}_{2 v}$ & 1.447 & 1.345 & 124.6 & 120.1 & 360.0 & 180.0 & 0.37 & 5.08 & 0.32 & 5.26 \\
& 1.447 & 1.345 & & 120.1 & 360.0 & 180.0 & 0.38 & 5.39 & & \\
\hline $\boldsymbol{S}_{1}$-Min $\boldsymbol{C}_{2}$ & 1.454 & 1.322 & 105.5 & 122.7 & 360.0 & 110.5 & $f^{f} 3.57$ & $f^{f} 4.37$ & 3.12 & 4.08 \\
& 1.454 & 1.322 & & 122.7 & 360.0 & 110.5 & $f_{3} 3.43$ & $f_{4} 4.35$ & & \\
\hline $\boldsymbol{C}_{2}$-CI & 1.458 & 1.331 & 96.8 & 122.8 & 359.9 & 108.3 & $f^{f} 4.47$ & $f^{f} 4.47$ & 3.96 & 4.26 \\
& 1.458 & 1.331 & & 122.8 & 359.9 & 108.3 & $f_{4} 4.22$ & $f_{4.34}$ & & \\
\hline $\boldsymbol{S}_{1}$-Min $\boldsymbol{C}_{1}$ & 1.423 & 1.293 & 121.4 & 123.4 & 360.0 & -179.7 & $f^{f} 3.12$ & $f^{f} 3.84$ & 2.61 & 2.98 \\
& 1.462 & 1.399 & & 116.6 & 336.4 & 76.1 & $f_{2.84}$ & $f_{3.99}$ & & \\
\hline $\boldsymbol{C}_{1}$-CI & 1.431 & 1.287 & 121.5 & 123.1 & 360.0 & -177.8 & ${ }^{f} 3.89$ & $f^{f} 3.89$ & ${ }^{g} \mathrm{CF}$ & ${ }^{g} \mathrm{CF}$ \\
& 1.467 & 1.423 & & 114.4 & 329.1 & 75.6 & $f_{3.49}$ & $f_{4.00}$ & & \\
\hline
\end{tabular}

PBE0

Geometries

\begin{tabular}{c|c|c|c|c|c|c|c|c|c|c}
\hline $\boldsymbol{S}_{0}$-trans & 1.387 & 1.317 & 118.7 & 125.6 & 360.0 & 180.0 & & & $\mathbf{0 . 0}$ & 5.45 \\
& 1.387 & 1.317 & & 125.6 & 360.0 & 180.0 & & & & \\
\hline
\end{tabular}

CIS

Geometries

\begin{tabular}{c|c|c|c|c|c|c|c|c|c|c}
\hline $\boldsymbol{S}_{1}-\operatorname{Min} \boldsymbol{C}_{2 v}$ & 1.426 & 1.331 & 125.7 & 121.1 & 360.0 & 180.0 & & & 0.19 & 5.27 \\
& 1.426 & 1.331 & & 121.1 & 360.0 & 180.0 & & & & \\
\hline $\boldsymbol{S}_{1}-\operatorname{Min} \boldsymbol{C}_{2}$ & 1.429 & 1.320 & 104.0 & 123.6 & 360.0 & 98.4 & & & 3.74 & 3.91 \\
& 1.429 & 1.320 & & 123.6 & 360.0 & 98.4 & & & & \\
\hline $\boldsymbol{S}_{1}-\operatorname{Min} \boldsymbol{C}_{1}$ & 1.441 & 1.289 & 122.0 & 123.3 & 360.0 & 179.2 & & & 2.09 & 3.09 \\
& 1.408 & 1.380 & & 121.8 & 344.4 & 86.3 & & & &
\end{tabular}

Notes: In $C_{1}$ geometries the moiety coplanar with the central $\mathrm{C}_{0}-\mathrm{H}$ group is the one without apices. a) for each method (CASSCF, CASMP2, TD-PBE0/PBE0) the energies are relative to the $S_{0}$ minimum value which is set to zero and for convenience is highlighted in boldface; b) $\beta=\mathrm{C}_{2}-\mathrm{C}_{0}-\mathrm{C}_{2}^{\prime}$; c) $\gamma=\mathrm{N}_{3}-\mathrm{C}_{2}-\mathrm{C}_{0}, \gamma^{\prime}=\mathrm{N}_{3}^{\prime}-\mathrm{C}_{2}^{\prime}-\mathrm{C}_{0}$; d) $\Omega N_{3}=$ sum of the bond angles with vertex in $\mathrm{N}_{3}, \Omega N_{3}^{\prime}=$ sum of the bond angles with vertex in $\mathrm{N}_{3}^{\prime}$; e) $\phi=\mathrm{N}_{3}-\mathrm{C}_{2}-\mathrm{C}_{0}$ $\left.\mathrm{C}_{2}^{\prime}, \phi^{\prime}=\mathrm{N}_{3}^{\prime}-\mathrm{C}_{2}^{\prime}-\mathrm{C}_{0}-\mathrm{C}_{2} ; \mathrm{f}\right)$ state average computation with equal weights for the two states; g) convergence failure. 
Table S2: $\boldsymbol{S}_{0}$-trans structure of H-TCY optimized at PBE0/6-31G(d) level

\begin{tabular}{rrrr}
\hline 1 & 0.000000 & 0.000000 & 2.486184 \\
6 & 0.000000 & 0.000000 & 1.398816 \\
6 & 0.000000 & 1.248071 & 0.781397 \\
6 & 0.000000 & -1.248071 & 0.781397 \\
7 & 0.000000 & 2.407772 & 1.475809 \\
6 & 0.000357 & 3.578621 & 0.732858 \\
6 & 0.001201 & 3.310779 & -0.639263 \\
16 & 0.001130 & 1.579551 & -0.927094 \\
7 & 0.000000 & -2.407772 & 1.475809 \\
6 & -0.000357 & -3.578621 & 0.732858 \\
6 & -0.001201 & -3.310779 & -0.639263 \\
16 & -0.001130 & -1.579551 & -0.927094 \\
1 & -0.000742 & 2.412663 & 2.487489 \\
1 & 0.000742 & -2.412663 & 2.487489 \\
6 & -0.000792 & 4.886286 & 1.210386 \\
6 & -0.000759 & 5.912632 & 0.275065 \\
6 & 0.000267 & 5.645092 & -1.098483 \\
6 & 0.001123 & 4.339033 & -1.574339 \\
1 & -0.001561 & 5.095992 & 2.275791 \\
1 & -0.001626 & 6.941802 & 0.619516 \\
1 & 0.000276 & 6.468092 & -1.805764 \\
1 & 0.001714 & 4.131561 & -2.639461 \\
6 & 0.000792 & -4.886286 & 1.210386 \\
6 & 0.000759 & -5.912632 & 0.275065 \\
6 & -0.000267 & -5.645092 & -1.098483 \\
6 & -0.001123 & -4.339033 & -1.574339 \\
1 & 0.001561 & -5.095992 & 2.275791 \\
1 & 0.001626 & -6.941802 & 0.619516 \\
1 & -0.000276 & -6.468092 & -1.805764 \\
1 & -0.001714 & -4.131561 & -2.639461
\end{tabular}


Table S3 $\boldsymbol{S}_{1}$-Min $\boldsymbol{C}_{2}$ structure ogf $\mathrm{H}$-TCY optimized at CIS/6-31G(d) level

\begin{tabular}{rrrr}
\hline 6 & 0.000000 & 0.000000 & 1.519591 \\
6 & 0.000000 & 1.237437 & 0.867991 \\
7 & 0.470482 & 2.390889 & 1.425491 \\
6 & 0.364450 & 3.510415 & 0.649891 \\
6 & -0.239851 & 3.239706 & -0.580909 \\
16 & -0.638552 & 1.545455 & -0.720009 \\
6 & 0.000000 & -1.237437 & 0.867991 \\
7 & -0.470482 & -2.390889 & 1.425491 \\
6 & -0.364450 & -3.510415 & 0.649891 \\
6 & 0.239851 & -3.239706 & -0.580909 \\
16 & 0.638552 & -1.545455 & -0.720009 \\
1 & 0.931915 & 2.392567 & 2.309891 \\
1 & -0.931915 & -2.392567 & 2.309891 \\
1 & 0.000000 & 0.000000 & 2.602091 \\
6 & 0.780257 & 4.804637 & 0.971691 \\
6 & 0.565219 & 5.795448 & 0.044891 \\
6 & -0.047711 & 5.526938 & -1.188909 \\
6 & -0.454278 & 4.251107 & -1.509709 \\
1 & 1.248082 & 5.014619 & 1.915791 \\
1 & 0.872164 & 6.799954 & 0.269491 \\
1 & -0.199124 & 6.326139 & -1.889509 \\
1 & -0.922099 & 4.041225 & -2.453409 \\
6 & -0.780257 & -4.804637 & 0.971691 \\
6 & -0.565219 & -5.795448 & 0.044891 \\
6 & 0.047711 & -5.526938 & -1.188909 \\
6 & 0.454278 & -4.251107 & -1.509709 \\
1 & -1.248082 & -5.014619 & 1.915791 \\
1 & -0.872164 & -6.799954 & 0.269491 \\
1 & 0.199124 & -6.326139 & -1.889509 \\
1 & 0.922099 & -4.041225 & -2.453409
\end{tabular}


Table S4 $\boldsymbol{S}_{1}$-Min structure of H-TCY optimized at CIS/6-31G(d) level

\begin{tabular}{rrrr}
\hline 6 & 0.033717 & -1.237854 & 0.505357 \\
6 & 1.316536 & -0.686001 & 0.302674 \\
7 & 2.462292 & -1.307070 & 0.553903 \\
6 & 3.607595 & -0.574215 & 0.288694 \\
6 & 3.294060 & 0.685856 & -0.202200 \\
16 & 1.561654 & 0.893159 & -0.316672 \\
6 & -1.146680 & -0.510070 & 0.177720 \\
7 & -2.014709 & -0.053570 & 1.164491 \\
6 & -3.305079 & 0.203422 & 0.719998 \\
6 & -3.525208 & -0.227990 & -0.579038 \\
16 & -2.053268 & -0.915443 & -1.272915 \\
1 & 2.487554 & -2.240580 & 0.911304 \\
1 & -1.651741 & 0.436576 & 1.951965 \\
1 & 0.006240 & -2.258552 & 0.875394 \\
6 & 4.926825 & -0.982400 & 0.465041 \\
6 & 5.912829 & -0.085282 & 0.132099 \\
6 & 5.603802 & 1.190487 & -0.366002 \\
6 & 4.299592 & 1.588985 & -0.538583 \\
1 & 5.162585 & -1.958873 & 0.845605 \\
1 & 6.942100 & -0.364879 & 0.254507 \\
1 & 6.401228 & 1.864076 & -0.616261 \\
1 & 4.062947 & 2.564103 & -0.920518 \\
6 & -4.324015 & 0.799743 & 1.446666 \\
6 & -5.560522 & 0.941341 & 0.843697 \\
6 & -5.783533 & 0.501054 & -0.457029 \\
6 & -4.762816 & -0.088837 & -1.181192 \\
1 & -4.159058 & 1.135552 & 2.454256 \\
1 & -6.362331 & 1.398651 & 1.393041 \\
1 & -6.752248 & 0.621039 & -0.904162 \\
1 & -4.927162 & -0.425518 & -2.187922
\end{tabular}


Table S5 CI structure of H-TCY optimized at CAS(6/6)/6-31G(d) level

\begin{tabular}{rrrr}
\hline $\mathrm{c}$ & 0.044427 & -1.299582 & 0.530664 \\
$\mathrm{c}$ & 1.336289 & -0.693266 & 0.296363 \\
$\mathrm{n}$ & 2.477509 & -1.270047 & 0.585112 \\
$\mathrm{c}$ & 3.612597 & -0.522464 & 0.293863 \\
$\mathrm{c}$ & 3.264402 & 0.701634 & -0.253874 \\
$\mathrm{~S}$ & 1.530840 & 0.851926 & -0.378356 \\
$\mathrm{c}$ & -1.179438 & -0.588456 & 0.168995 \\
$\mathrm{n}$ & -1.972282 & -0.107415 & 1.252103 \\
$\mathrm{c}$ & -3.252545 & 0.229657 & 0.716803 \\
$\mathrm{c}$ & -3.522606 & -0.274264 & -0.527871 \\
$\mathrm{~S}$ & -2.172816 & -1.251246 & -1.161882 \\
$\mathrm{~h}$ & 2.523518 & -2.186413 & 0.987035 \\
$\mathrm{~h}$ & -2.076199 & -0.769394 & 2.003148 \\
$\mathrm{~h}$ & 0.052877 & -2.283919 & 0.977505 \\
$\mathrm{c}$ & 4.940246 & -0.891168 & 0.498881 \\
$\mathrm{c}$ & 5.901714 & 0.017372 & 0.133976 \\
$\mathrm{c}$ & 5.558657 & 1.262208 & -0.421530 \\
$\mathrm{c}$ & 4.247888 & 1.619519 & -0.622427 \\
$\mathrm{~h}$ & 5.198620 & -1.843425 & 0.923360 \\
$\mathrm{~h}$ & 6.937751 & -0.225558 & 0.274869 \\
$\mathrm{~h}$ & 6.339623 & 1.946482 & -0.693911 \\
$\mathrm{~h}$ & 3.988849 & 2.570846 & -1.047094 \\
$\mathrm{c}$ & -4.201370 & 1.005460 & 1.387824 \\
$\mathrm{c}$ & -5.415051 & 1.243077 & 0.773730 \\
$\mathrm{c}$ & -5.681014 & 0.727465 & -0.504948 \\
$\mathrm{c}$ & -4.742217 & -0.026202 & -1.163003 \\
$\mathrm{~h}$ & -3.978169 & 1.408141 & 2.358650 \\
$\mathrm{~h}$ & -6.157934 & 1.837651 & 1.272111 \\
$\mathrm{~h}$ & -6.626073 & 0.929991 & -0.973914 \\
$\mathrm{~h}$ & -4.939696 & -0.418996 & -2.143135
\end{tabular}


Table S6 $\boldsymbol{S}_{0}$-cis structure of H-TCY optimized at PBE0/6-31G(d) level

\begin{tabular}{rrrr}
\hline 6 & -0.040006 & -1.451239 & 0.028052 \\
6 & -1.259164 & -0.785273 & 0.010519 \\
7 & -2.450734 & -1.419569 & 0.087557 \\
6 & -3.586839 & -0.624332 & 0.053795 \\
6 & -3.264832 & 0.731916 & -0.057039 \\
16 & -1.522336 & 0.931744 & -0.140096 \\
6 & 1.241104 & -0.896060 & 0.017330 \\
7 & 1.599171 & 0.402454 & 0.084329 \\
6 & 2.965254 & 0.665566 & 0.064563 \\
6 & 3.712885 & -0.512425 & -0.030253 \\
16 & 2.653141 & -1.905000 & -0.093480 \\
1 & -2.500426 & -2.425868 & 0.182101 \\
1 & 0.927207 & 1.143555 & 0.236501 \\
1 & -0.084684 & -2.535929 & 0.044607 \\
6 & -4.913253 & -1.040739 & 0.121078 \\
6 & -5.899523 & -0.063723 & 0.075541 \\
6 & -5.575516 & 1.292456 & -0.034901 \\
6 & -4.250542 & 1.709178 & -0.103811 \\
1 & -5.167509 & -2.092895 & 0.206643 \\
1 & -6.941648 & -0.362295 & 0.126881 \\
1 & -6.367519 & 2.033489 & -0.067988 \\
1 & -3.998682 & 2.760983 & -0.191219 \\
6 & 3.582188 & 1.910983 & 0.129004 \\
6 & 4.970017 & 1.943137 & 0.094944 \\
6 & 5.720926 & 0.766233 & -0.001312 \\
6 & 5.102586 & -0.476945 & -0.064818 \\
1 & 3.000009 & 2.824730 & 0.202441 \\
1 & 5.478946 & 2.900414 & 0.143601 \\
1 & 6.804403 & 0.822023 & -0.026876 \\
1 & 5.686254 & -1.388708 & -0.138831
\end{tabular}


Table S7 $\boldsymbol{S}_{0}$-trans structure of Et-TCY optimized at PBE0/6-31G(d) level

\begin{tabular}{rrrr}
\hline 6 & 0.000000 & 0.000000 & 0.840488 \\
6 & -0.001202 & 1.253211 & 0.223455 \\
7 & 0.074046 & 2.426374 & 0.903456 \\
6 & 0.000000 & 3.570455 & 0.108744 \\
6 & 0.011561 & 4.900253 & 0.530480 \\
6 & -0.055091 & 5.894570 & -0.437141 \\
6 & -0.134669 & 5.583506 & -1.797887 \\
6 & -0.154606 & 4.260824 & -2.222074 \\
6 & -0.089971 & 3.266875 & -1.252808 \\
16 & -0.113652 & 1.536732 & -1.491721 \\
6 & 0.001202 & -1.253211 & 0.223455 \\
7 & -0.074046 & -2.426374 & 0.903456 \\
6 & 0.000000 & -3.570455 & 0.108744 \\
6 & -0.011561 & -4.900253 & 0.530480 \\
6 & 0.055091 & -5.894570 & -0.437141 \\
6 & 0.134669 & -5.583506 & -1.797887 \\
6 & 0.154606 & -4.260824 & -2.222074 \\
6 & 0.089971 & -3.266875 & -1.252808 \\
16 & 0.113652 & -1.536732 & -1.491721 \\
6 & 0.190342 & 2.508187 & 2.359276 \\
6 & -1.160890 & 2.519136 & 3.057625 \\
1 & 0.817484 & 1.677142 & 2.692683 \\
1 & 0.757472 & 3.415561 & 2.580002 \\
1 & -1.018256 & 2.592079 & 4.139982 \\
1 & -1.762633 & 3.374068 & 2.735184 \\
1 & -1.729300 & 1.607872 & 2.846670 \\
6 & -0.190342 & -2.508187 & 2.359276 \\
6 & 1.160890 & -2.519136 & 3.057625 \\
1 & -0.817484 & -1.677142 & 2.692683 \\
1 & -0.757472 & -3.415561 & 2.580002 \\
1 & 1.018256 & -2.592079 & 4.139982 \\
1 & 1.762633 & -3.374068 & 2.735184 \\
1 & 1.729300 & -1.607872 & 2.846670 \\
1 & 0.061965 & 5.164141 & 1.581414 \\
1 & -0.047765 & 6.934051 & -0.124896 \\
1 & -0.185398 & 6.381114 & -2.531931 \\
1 & -0.220926 & 4.011257 & -3.276170 \\
1 & -0.061965 & -5.164141 & 1.581414 \\
1 & 0.047765 & -6.934051 & -0.124896 \\
1 & 0.185398 & -6.381114 & -2.531931 \\
1 & 0.220926 & -4.011257 & -3.276170 \\
1 & 0.000000 & 0.000000 & 1.921849
\end{tabular}


Table S8 $\boldsymbol{S}_{1}$-Min $\boldsymbol{C}_{2}$ structure of H-TCY optimized at CIS/6-31G(d) level

\begin{tabular}{rrrr}
\hline 6 & 0.000000 & 0.000000 & 0.981008 \\
6 & 0.000000 & 1.235532 & 0.315814 \\
7 & 0.506176 & 2.404813 & 0.814638 \\
6 & 0.291141 & 3.494500 & 0.005848 \\
6 & 0.673219 & 4.820151 & 0.246711 \\
6 & 0.361819 & 5.770095 & -0.696395 \\
6 & -0.320575 & 5.441343 & -1.875621 \\
6 & -0.701524 & 4.141679 & -2.118909 \\
6 & -0.389392 & 3.173364 & -1.172324 \\
16 & -0.737024 & 1.470762 & -1.243060 \\
6 & 0.000000 & -1.235532 & 0.315814 \\
7 & -0.506176 & -2.404813 & 0.814638 \\
6 & -0.291141 & -3.494500 & 0.005848 \\
6 & -0.673219 & -4.820151 & 0.246711 \\
6 & -0.361819 & -5.770095 & -0.696395 \\
6 & 0.320575 & -5.441343 & -1.875621 \\
6 & 0.701524 & -4.141679 & -2.118909 \\
6 & 0.389392 & -3.173364 & -1.172324 \\
16 & 0.737024 & -1.470762 & -1.243060 \\
6 & 1.238066 & 2.486793 & 2.082191 \\
6 & 0.343778 & 2.851824 & 3.263941 \\
1 & 1.722756 & 1.534250 & 2.239824 \\
1 & 2.025536 & 3.215763 & 1.955019 \\
1 & 0.942035 & 2.911748 & 4.166428 \\
1 & -0.138632 & 3.809927 & 3.112765 \\
1 & -0.430456 & 2.108652 & 3.419226 \\
6 & -1.238066 & -2.486793 & 2.082191 \\
6 & -0.343778 & -2.851824 & 3.263941 \\
1 & -1.722756 & -1.534250 & 2.239824 \\
1 & -2.025536 & -3.215763 & 1.955019 \\
1 & -0.942035 & -2.911748 & 4.166428 \\
1 & 0.138632 & -3.809927 & 3.112765 \\
1 & 0.430456 & -2.108652 & 3.419226 \\
1 & 1.187195 & 5.099910 & 1.145272 \\
1 & 0.646254 & 6.791170 & -0.522162 \\
1 & -0.547208 & 6.208943 & -2.591227 \\
1 & -1.224975 & 3.877986 & -3.019033 \\
1 & -1.187195 & -5.099910 & 1.145272 \\
1 & -0.646254 & -6.791170 & -0.522162 \\
1 & 0.547208 & -6.208943 & -2.591227 \\
1 & 1.224975 & -3.877986 & -3.019033 \\
1 & 0.000000 & 0.000000 & 2.059658
\end{tabular}


Table S9 $\boldsymbol{S}_{1}$-Min structure of Et-TCY optimized at CIS/6-31G(d) level

\begin{tabular}{rrrr}
\hline 6 & 0.002245 & 0.936398 & -0.272248 \\
6 & 1.270886 & 0.342246 & -0.082001 \\
7 & 2.459074 & 0.936513 & -0.149493 \\
6 & 3.527918 & 0.067011 & 0.059896 \\
6 & 4.888971 & 0.378615 & 0.040242 \\
6 & 5.783492 & -0.636745 & 0.279140 \\
6 & 5.356034 & -1.947980 & 0.534143 \\
6 & 4.018291 & -2.257665 & 0.550199 \\
6 & 3.106015 & -1.233073 & 0.308532 \\
16 & 1.369664 & -1.340609 & 0.252652 \\
6 & -1.185120 & 0.142495 & -0.221319 \\
7 & -2.158412 & 0.295494 & 0.758864 \\
6 & -3.429160 & -0.092861 & 0.355826 \\
6 & -4.577585 & -0.136676 & 1.137922 \\
6 & -5.763006 & -0.547637 & 0.553595 \\
6 & -5.822845 & -0.914272 & -0.784900 \\
6 & -4.680706 & -0.873641 & -1.565233 \\
6 & -3.497064 & -0.462890 & -0.982496 \\
16 & -1.914206 & -0.346156 & -1.744995 \\
6 & 2.653821 & 2.371181 & -0.443520 \\
6 & 2.821120 & 2.638338 & -1.934840 \\
1 & 1.813120 & 2.908914 & -0.035456 \\
1 & 3.520001 & 2.687563 & 0.116075 \\
1 & 2.980541 & 3.698529 & -2.092191 \\
1 & 3.672147 & 2.103687 & -2.338758 \\
1 & 1.938248 & 2.342791 & -2.489423 \\
6 & -1.832711 & 0.618307 & 2.142558 \\
6 & -2.100125 & 2.077623 & 2.499449 \\
1 & -2.401625 & -0.040800 & 2.785590 \\
1 & -0.792169 & 0.373318 & 2.303246 \\
1 & -1.860132 & 2.253127 & 3.542719 \\
1 & -1.495828 & 2.746293 & 1.895873 \\
1 & -3.139909 & 2.338126 & 2.343160 \\
1 & 5.240056 & 1.371792 & -0.159116 \\
1 & 6.835159 & -0.420485 & 0.268101 \\
1 & 6.084047 & -2.715707 & 0.715562 \\
1 & 3.681737 & -3.259291 & 0.741444 \\
1 & -4.561124 & 0.148059 & 2.172098 \\
1 & -6.654268 & -0.579980 & 1.152574 \\
1 & -6.754134 & -1.229773 & -1.215988 \\
1 & -4.712339 & -1.158018 & -2.600790 \\
1 & -0.028319 & 1.983403 & -0.542486
\end{tabular}


Table S10: $\boldsymbol{S}_{0}$-cis structure of Et-TCY optimized at PBE0/6-31G(d) level

\begin{tabular}{rrrr}
\hline 6 & -0.047373 & 1.245401 & -0.131828 \\
6 & -1.216796 & 0.490192 & -0.176049 \\
7 & -2.466982 & 1.020778 & -0.128786 \\
6 & -3.503899 & 0.092842 & -0.252426 \\
6 & -4.875419 & 0.339101 & -0.199111 \\
6 & -5.743034 & -0.736900 & -0.343529 \\
6 & -5.266057 & -2.036101 & -0.533606 \\
6 & -3.899897 & -2.288494 & -0.577435 \\
6 & -3.033793 & -1.211729 & -0.435507 \\
16 & -1.285515 & -1.235230 & -0.447896 \\
6 & 1.282963 & 0.785122 & -0.184870 \\
7 & 1.841744 & -0.284419 & 0.422551 \\
6 & 3.188187 & -0.491789 & 0.095446 \\
6 & 4.001102 & -1.542707 & 0.518798 \\
6 & 5.324600 & -1.554670 & 0.099234 \\
6 & 5.835490 & -0.543526 & -0.721419 \\
6 & 5.028174 & 0.502844 & -1.148300 \\
6 & 3.700222 & 0.511794 & -0.733793 \\
16 & 2.445689 & 1.650180 & -1.147638 \\
6 & -2.718519 & 2.442594 & 0.109080 \\
6 & -2.661490 & 2.807345 & 1.584151 \\
1 & -1.999213 & 3.019874 & -0.478267 \\
1 & -3.696786 & 2.667892 & -0.319560 \\
1 & -2.863530 & 3.875225 & 1.709418 \\
1 & -3.409276 & 2.248658 & 2.154894 \\
1 & -1.674481 & 2.595300 & 2.006094 \\
6 & 1.265869 & -0.933321 & 1.611318 \\
6 & 1.924152 & -0.449985 & 2.895281 \\
1 & 0.202818 & -0.705926 & 1.633188 \\
1 & 1.358905 & -2.015850 & 1.480749 \\
1 & 1.460159 & -0.958125 & 3.746056 \\
1 & 2.996461 & -0.658953 & 2.919214 \\
1 & 1.778367 & 0.627105 & 3.021271 \\
1 & -5.269917 & 1.336981 & -0.041047 \\
1 & -6.812947 & -0.558659 & -0.304093 \\
1 & -5.965655 & -2.858146 & -0.644934 \\
1 & -3.521466 & -3.295469 & -0.721319 \\
1 & 3.617010 & -2.335641 & 1.152027 \\
1 & 5.971632 & -2.366964 & 0.414653 \\
1 & 6.874012 & -0.577842 & -1.034671 \\
1 & 5.418450 & 1.284590 & -1.791898 \\
1 & -0.157551 & 2.314152 & -0.282191
\end{tabular}

